\title{
PANORAMA DA DIARREIA E GASTROENTERITES ENTRE CRIANÇAS BRASILEIRAS NA ÚLTIMA DÉCADA
}

\section{PANORAMA OF DIARRHEA AND GASTROENTERITIS AMONG BRAZILIAN CHILDREN IN LAST DECADE}

\author{
Samylla Maira Costa Siqueira ${ }^{1}$, Rafaela Mainarte Costa Franco ${ }^{2}$, Climene Laura de Camargo ${ }^{1}$, \\ Jacqueline Couto Nascimento ${ }^{2}$, Iraci Alcântara Mariano² \\ Universidade Federal da Bahia ${ }^{1}$, Centro Universitário Jorge Amado ${ }^{2}$
}

\begin{abstract}
Objectives: To describe the panorama of diarrhea and gastroenteritis among Brazilian children in last decade. Methods: A time-series ecological study, carried out in March 2019 with secondary data from DATASUS. The target population consisted of people in the 0-5 age group, both male and female, affected by diarrhea and gastroenteritis of infectious origin, from 20092018. Results: In the the studied period, occurred 647.343 hospitalizations and 789 deaths of children under 5 years of age by diarrhea and gastroenteritis of infectious origin. Male was more affected in both, hospitalizations, and deaths. In relation to region, northeast presented the highest number of hospitalizations and deaths and South the smaller. Concerning race/color, blacks were more affected. In general, the cases of diarrhea and gastroenteritis in Brazil have dropped. About the age group, although children aged 1-5 years of age were the most affected, the highest number of deaths were identified among under 1 years of age. Conclusions: the higher number of hospitalizations and deaths occurred among black children from region northeast, reflecting the need to intensify the measures to prevent and care in relation to diarrhea and gastroenteritis among these people.
\end{abstract}

Key words: Diarrhea, Child Health, Epidemiology.
Resumo

Objetivos: Descrever o panorama da diarreia $e$ gastroenterites entre crianças brasileiras na última década. Métodos: Estudo ecológico de série temporal, realizado em março de 2019 com dados secundários do DATASUS. A população de estudo foi composta de indivíduos na faixa etária de 0-5 anos, de ambos os sexos, acometidos por diarreia e gastroenterites de origem infecciosa, no período de 2009-2018. Resultados: No período do estudo, ocorreram 647.343 internações e 789 óbitos de crianças menores de 5 anos de idade por diarreia e gastroenterites de origem infecciosa. $O$ sexo masculino foi o mais acometido em ambos os casos. No que concerne à região, o Nordeste teve o maior número de internações e óbitos e o Sul o menor. Quanto ao recorte raça/cor, os negros foram os mais acometidos. De forma geral, houve queda nos casos de diarreia e gastroenterites no Brasil. Em relação à faixa etária, embora as crianças entre 1-5 anos fossem as mais acometidas, o maior número de óbitos foi identificado entre aquelas menores de 1 ano de idade. Conclusões: O maior número de internações e óbitos ocorreu entre crianças negras da Região Nordeste, refletindo a necessidade de intensificação de medidas de prevenção e cuidados no que diz respeito à diarreia e gastroenterites entre estas pessoas.

Palavras chave: Diarreia, Saúde da Criança, Epidemiologia. 
Introdução

A diarreia é provocada pela presença de microrganismos de diferentes etiologias, caracterizando-se como uma síndrome em que há aumento do número de evacuações, com alteração na consistência e aspecto das fezes. Os efeitos fisiológicos mais importantes são desidratação e desnutrição relacionadas aos distúrbios hidroeletrolíticos, sendo geralmente acompanhadas de náuseas, vômitos, cólicas abdominais e febre, que podem se agravar de acordo à forma que a diarreia se apresenta e a duração da mesma, em geral, de 2 a 14 dias $^{1}$.

Por conta de suas consequências, a diarreia tem grande importância epidemiológica na infância, especialmente entre crianças menores de cinco anos de idade, quando é caracterizada como uma das doenças prevalentes na infância. Dados do Departamento de Informática do Sistema Único de Saúde (DATASUS) demonstram ser a diarreia, juntamente com outras gastroenterites, uma importante causa de internação entre crianças com idade inferior a cinco anos, de forma que no ano de 2018 a diarreia figurou como a 1a causa de internação no Brasil, com gasto total de $\mathrm{R} \$ 1$ 17.138.220,09 para o sistema de saúde brasileiro ${ }^{2}$.

Dada a relevância dos óbitos precoces de crianças por diarreia e outros agravos, a Organização das Nações Unidas (ONU) estabeleceu os Objetivos de Desenvolvimento do Milênio (ODM), sendo a 4a meta a redução da mortalidade infantil. Dados revelam que o Brasil tem conseguido alcançar tal objetivo, de modo que a mortalidade de crianças menores de 1 ano de idade caiu de 47,1 óbitos por mil nascimentos em 1990 para 19 em 2008. Destaca-se, contudo, que esta queda não é horizontal em todo o Brasil, de forma que em localidades mais precárias as crianças têm mais que o dobro de chances de morrer por esta causa que aquelas que habitam locais mais abastados ${ }^{3}$.

Corroborando tal assertiva, dados do DATASUS revelam que foi o Nordeste a região brasileira onde ocorreram os maiores índices de internações e óbitos de indivíduos menores de 1 ano de idade por diarreia no ano de 2018, evidenciando a vulnerabilidade de crianças que vivem em locais precários no que concerne às doenças de veiculação hídrica e suas consequências ${ }^{2}$.

Frente à realidade supracitada, é imprescindível a realização de estudos que revelem a tendência das doenças diarreicas, dado o potencial destas pesquisas de fundamentar ações voltadas para o combate deste problema na infância e, por consequência, reduzir a morbimortalidade de crianças por conta da diarreia. Ademais, ao revelar as diferenças regionais na ocorrência deste problema, podemse direcionar ações estratégicas para áreas mais precárias, favorecendo a equidade em saúde e a redução dos óbitos de crianças mais pobres por desnutrição e/ou desidratação.

Diante do exposto, o objetivo deste estudo é descrever o panorama da diarreia e gastroenterites entre crianças brasileiras na última década.

\section{Método}

Estudo ecológico de série temporal realizado em março de 2019 a partir de dados secundários extraídos do DATASUS. Considerando-se o objeto de estudo proposto "panorama da diarreia e gastroenterites entre crianças brasileiras na última década", a questão de pesquisa que norteou a busca dos dados foi: qual é o panorama da diarreia e gastroenterites entre crianças na última década no Brasil?

A população de estudo foi composta de indivíduos de na faixa etária de 0-5 anos, de ambos os sexos, que foram internados ou morreram no período de 2009-2018 por diarreia e gastroenterites de origem infecciosa (Lista Morbidade CID-10). Os dados foram tabulados considerando-se cinco variáveis: 1) Sexo; 2) Ano; 3) Faixa etária; 4) Região brasileira; e 5) Raça/cor.

Para análise dos dados, foi utilizado o programa Microsoft Excel 2010, sendo os resultados apresentados sob a forma de frequências absolutas e relativas a partir de 4 gráficos e 1 tabela.

Por terem sido utilizadas informações de fontes secundárias, não foi necessária submissão do estudo à Plataforma Brasil e aprovação por comitê de ética em pesquisa.

\section{Resultados}

Para identificar a tendência da diarreia e gastroenterites entre crianças brasileiras na última década, foram analisados 647.343 internações e 789 óbitos de crianças menores de cinco anos de idade por esta causa no período de 2009-2018. Conforme pode ser observado no Gráfico 1, as internações por esta causa ocorreram principalmente entre as crianças de 1 a 4 anos de idade $(72,2 \%)$, seguidas daquelas menores de 1 ano $(27,77 \%)$, sendo sexo masculino o mais acometido $(53,71 \%)$ de forma geral. 
Gráfico 1 - Internações de crianças menores de 5 anos de idade por diarreia e outras gastroenterites ( $n=647.343$ ) no Brasil no período de 2009-2018 quanto ao sexo e faixa etária.

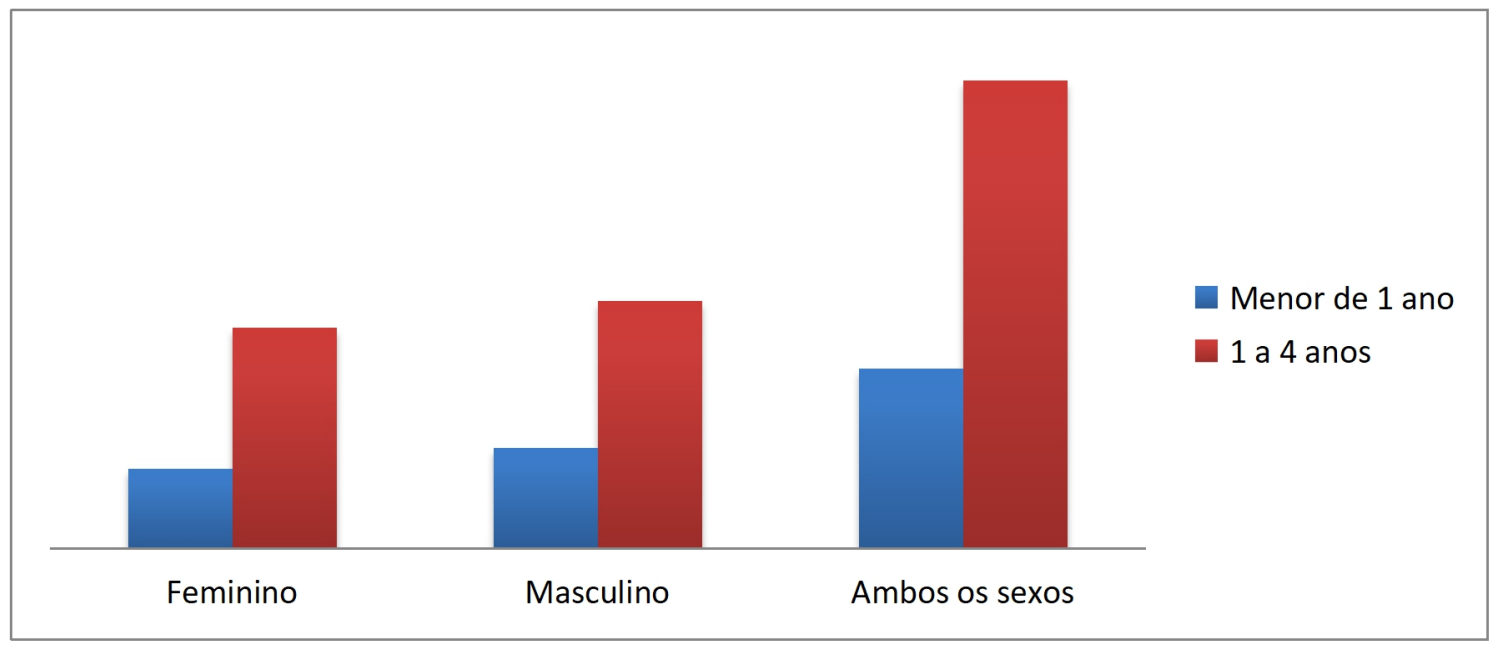

Fonte: Ministério da Saúde - Sistema de Informações Hospitalares do SUS (SIH/SUS).

Ao direcionar a análise por localidade, pode-se constatar que as regiões brasileiras não são afetadas de modo linear, tendo sido o Nordeste $(42,90 \%)$ a que mais apresentou casos de internações por diarreia e gastroenterites entre as crianças menores de 5 anos de idade no período em questão em todos os anos analisados (Gráfico 2), seguida do Norte $(23,09 \%)$, Sudeste $(20,09 \%)$, Centro-Oeste $(7,08 \%)$ e Sul $(6,82 \%)$, respectivamente.

Gráfico 2 - Internação de crianças menores de 5 anos de idade por diarreia e outras gastroenterites no Brasil ( $n=647.343$ ) no período de 2009-2018 quanto ao ano e região brasileira.

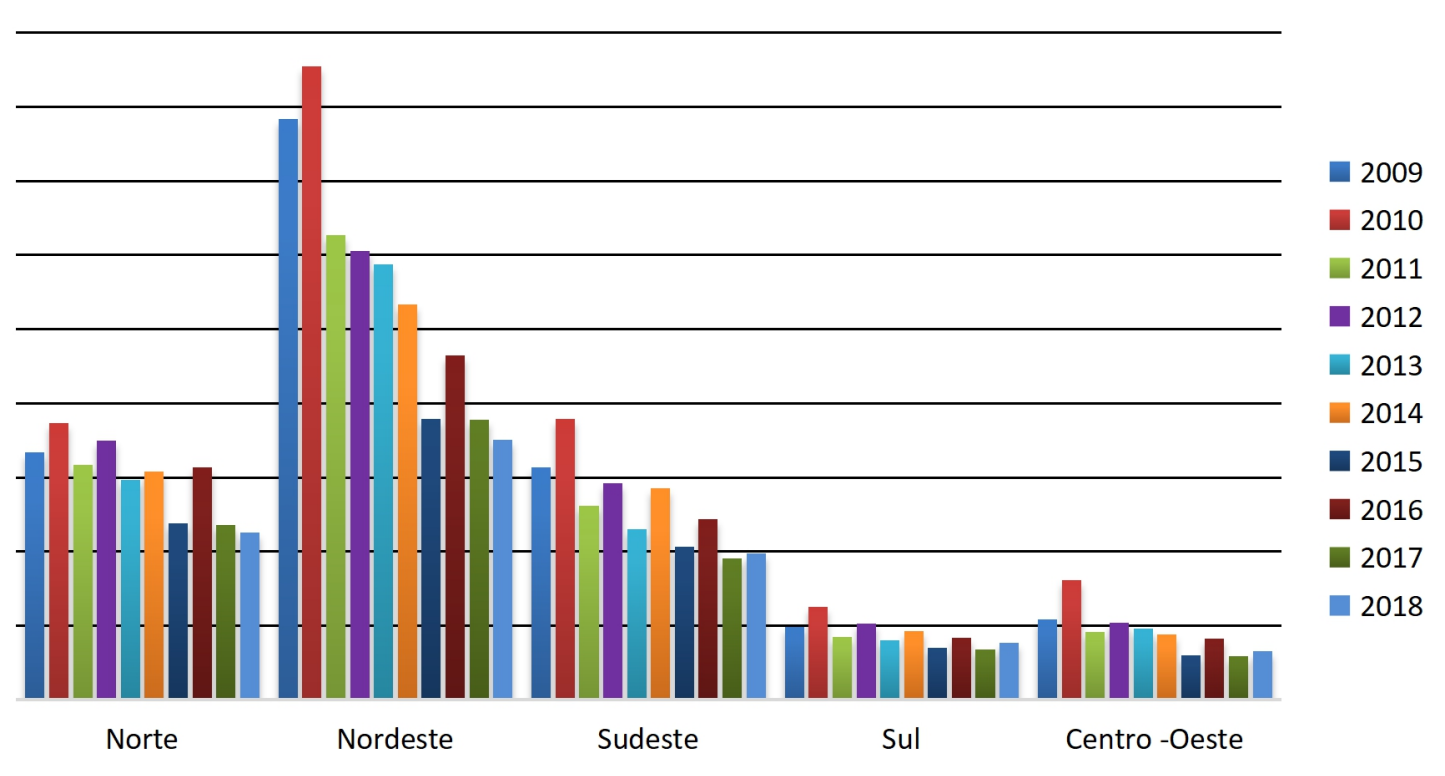

Fonte: Ministério da Saúde - Sistema de Informações Hospitalares do SUS (SIH/SUS).

No que concerne ao recorte ano e raça/cor, de acordo com a visualização do Gráfico 3 podese perceber que as crianças mais afetadas foram as negras $(45,82 \%)$, tendo sido $44,45 \%$ as pardas e $1,36 \%$ as pretas. Depois dos indivíduos negros, os mais acometidos foram os brancos $(17,98 \%)$, indígenas (1,56\%) e amarelos (0,40\%). Cabe destacar que houve uma quantidade significativa de casos de crianças internadas por doença diarreica sem identificação da variável raça/cor $(34,22 \%)$. 
Gráfico 3 - Internação de crianças menores de 5 anos de idade por diarreia e outras gastroenterites no Brasil $(n=647.343$ ) no período de $2009-2018$ quanto ao ano e raça/cor.

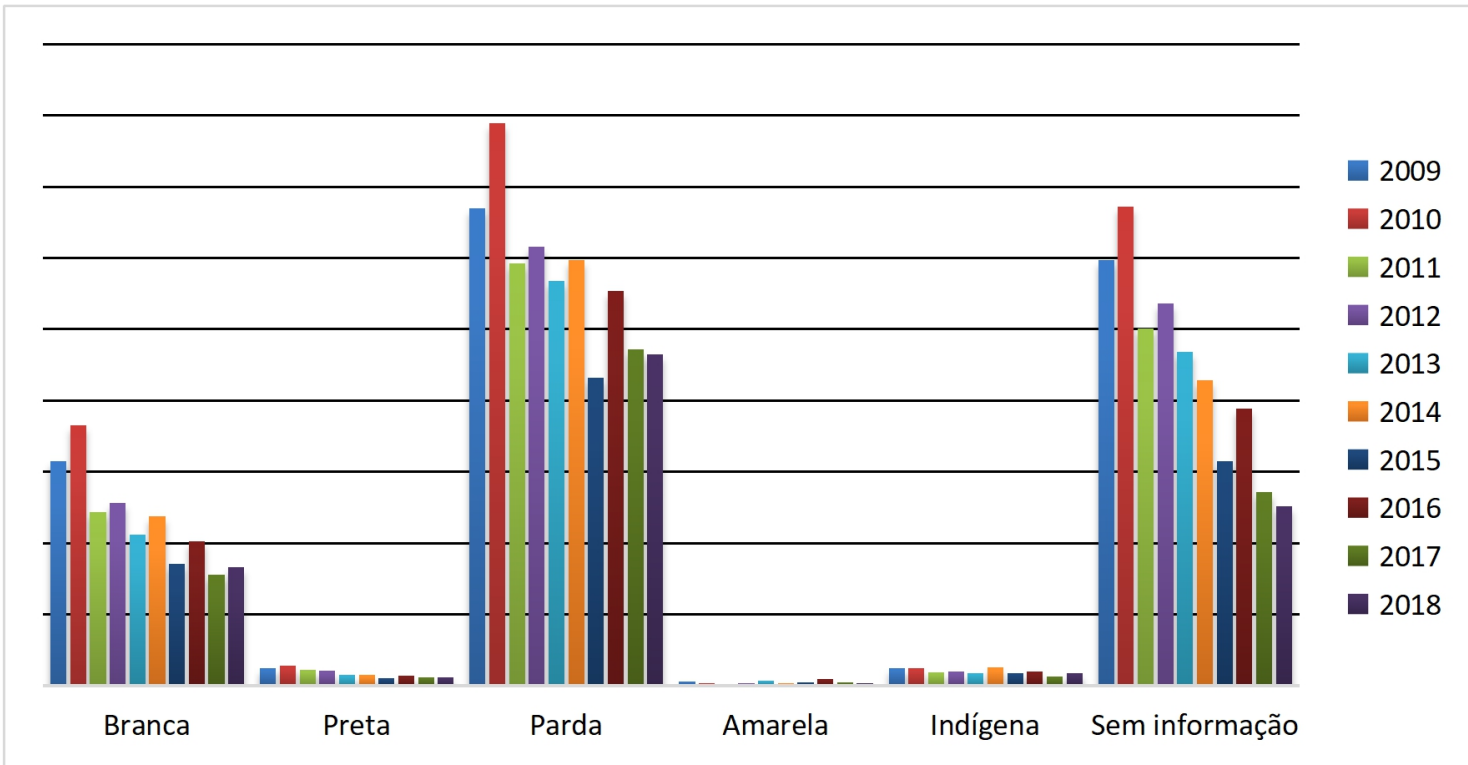

Fonte: Ministério da Saúde - Sistema de Informações Hospitalares do SUS (SIH/SUS).

No que diz respeito aos óbitos por ano quanto às regiões, conforme ilustrado pelo Gráfico 4, o ano de 2009 foi o que registrou o maior número de mortes de crianças brasileiras por conta da diarreia e outras gastroenterites $(14,32 \%)$ e no ano de 2015 a menor, respectivamente com (6,84\%). Pode-se perceber ainda que o Nordeste foi a região que apresentou o maior número de casos de óbitos $(44,35 \%)$, seguido do Norte (28,39\%), Sudeste $(17,23 \%)$, Centro-Oeste $(6,97 \%)$ e Sul $(3,04 \%)$.

Gráfico 4 - Óbitos de crianças menores de 5 anos de idade por diarreia e outras gastroenterites no Brasil (n=789) no período de 2009-2018 quanto ao ano e região brasileira.

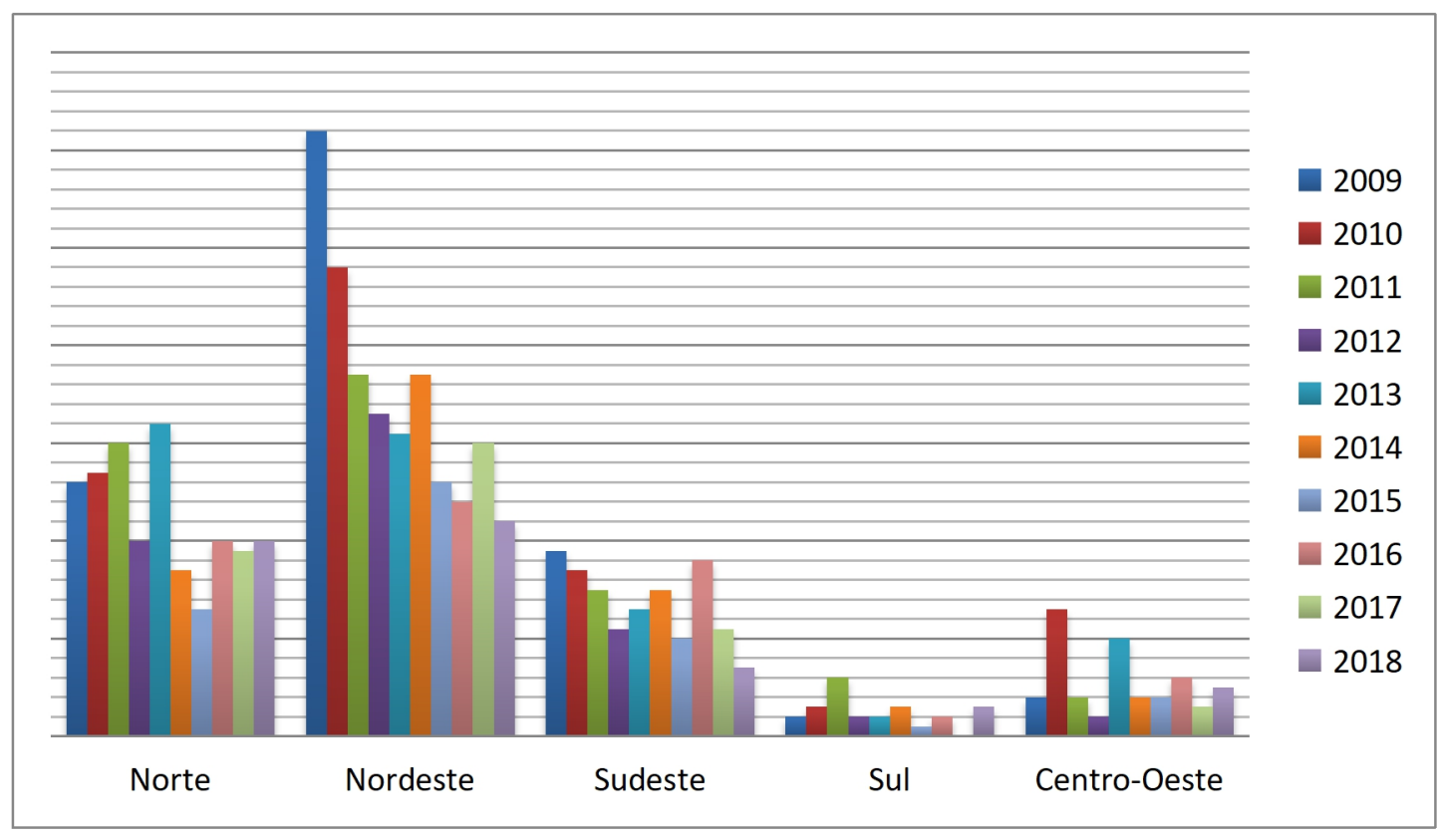

Fonte: Ministério da Saúde - Sistema de Informações Hospitalares do SUS (SIH/SUS). 
Na Tabela 1 são apresentados os dados concernentes aos óbitos. Quanto à faixa etária, ao contrário do que se observou nas internações, foram a óbito principalmente as crianças menores de 1 ano (72,75\%), tendo sido o sexo masculino $(55,64 \%)$ aquele que mais apresentou casos de morte por este problema, assim como ocorreu com as internações.

Ao destrinchar os dados por região brasileira (Tabela 1), constata-se que os óbitos seguem o mesmo padrão das internações, uma vez que a região mais afetada foi o Nordeste $(44,35 \%)$, seguido do Norte $(28,39 \%)$, Sudeste $(17,23 \%)$, Centro-Oeste $(6,97 \%)$ e Sul $(3,04 \%)$. No que concerne à raça/cor mais afetada pelos óbitos (Tabela 1), sobressaíram os indivíduos negros (37\%), seguidos dos brancos $(11,91 \%)$, indígenas $(10,51 \%)$ e amarelos $(0,50 \%)$. As crianças sem informação registraram $40,05 \%$ dos casos.

Tabela 1 - Óbitos de crianças menores de 5 anos de idade por diarreia e outras gastroenterites no Brasil ( $n=789)$ no período de 2009-2018 quanto ao ano, faixa etária, sexo, região brasileira e raça/cor.

\begin{tabular}{|c|c|c|c|c|c|c|c|c|c|c|c|}
\hline Variáveis & $\begin{array}{l}2009 \\
\text { N (\%) } \\
\end{array}$ & $\begin{array}{l}2010 \\
\text { N (\%) } \\
\end{array}$ & $\begin{array}{l}2011 \\
\text { N (\%) } \\
\end{array}$ & $\begin{array}{l}2012 \\
\text { N (\%) } \\
\end{array}$ & $\begin{array}{l}2013 \\
\text { N (\%) } \\
\end{array}$ & $\begin{array}{l}2014 \\
\text { N (\%) } \\
\end{array}$ & $\begin{array}{l}2015 \\
\text { N (\%) } \\
\end{array}$ & $\begin{array}{l}2016 \\
\text { N (\%) } \\
\end{array}$ & $\begin{array}{l}2017 \\
\text { N (\%) } \\
\end{array}$ & $\begin{array}{l}2018 \\
\text { N (\%) } \\
\end{array}$ & TOTAL \\
\hline \multicolumn{12}{|l|}{ Faixa etária } \\
\hline$<1$ & 81 & 71 & 71 & 46 & 64 & 64 & 39 & 51 & 53 & 34 & 574 \\
\hline $1-4$ & 32 & 37 & 21 & 22 & 24 & 12 & 15 & 19 & 10 & 23 & 215 \\
\hline Total & 113 & 108 & 92 & 68 & 88 & 76 & 54 & 70 & 63 & 57 & 789 \\
\hline \multicolumn{12}{|l|}{ Sexo } \\
\hline Masculino & 48 & 62 & 59 & 40 & 46 & 46 & 32 & 37 & 33 & 36 & 439 \\
\hline Feminino & 65 & 46 & 33 & 28 & 42 & 30 & 22 & 33 & 30 & 21 & 350 \\
\hline Total & 113 & 108 & 92 & 68 & 88 & 76 & 54 & 70 & 63 & 57 & 789 \\
\hline \multicolumn{12}{|l|}{ Região } \\
\hline Norte & 26 & 27 & 30 & 20 & 32 & 17 & 13 & 20 & 19 & 20 & 224 \\
\hline Nordeste & 62 & 48 & 37 & 33 & 31 & 37 & 26 & 24 & 30 & 22 & 350 \\
\hline Sudeste & 19 & 17 & 15 & 11 & 13 & 15 & 10 & 18 & 11 & 7 & 136 \\
\hline Sul & 2 & 3 & 6 & 2 & 2 & 3 & 1 & 2 & - & 3 & 24 \\
\hline Centro-Oeste & 4 & 13 & 4 & 2 & 10 & 4 & 4 & 6 & 3 & 5 & 55 \\
\hline Total & 113 & 108 & 92 & 68 & 88 & 76 & 54 & 70 & 63 & 57 & 789 \\
\hline \multicolumn{12}{|l|}{ Raça/Cor } \\
\hline Branca & 12 & 14 & 10 & 7 & 10 & 11 & 8 & 7 & 6 & 9 & 94 \\
\hline Preta & - & - & - & - & 1 & - & - & 1 & 1 & - & 3 \\
\hline Parda & 35 & 38 & 33 & 18 & 32 & 34 & 16 & 28 & 28 & 27 & 289 \\
\hline Amarela & - & - & 1 & 1 & - & - & - & - & - & 2 & 4 \\
\hline Indígena & 13 & 12 & 7 & 8 & 9 & 8 & 5 & 8 & 7 & 6 & 83 \\
\hline Sem informação & 53 & 44 & 41 & 34 & 36 & 23 & 25 & 26 & 21 & 13 & 316 \\
\hline Total & 113 & 108 & 92 & 68 & 88 & 76 & 54 & 70 & 63 & 57 & 789 \\
\hline
\end{tabular}

Fonte: Ministério da Saúde - Sistema de Informações Hospitalares do SUS (SIH/SUS).

\section{DISCUSSÃO}

A Organização Mundial de Gastroenterologia (WGO) ${ }^{4}$ refere que nas últimas três décadas, a taxa de mortalidade infantil relacionada às doenças diarreicas foi reduzida nos países em desenvolvimento e atribui isto a diversos fatores. No contexto do Brasil, tal evidência é comprovada ao se observar os dados relativos aos óbitos de crianças menores de 5 anos de idade por conta da diarreia e gastroenterites, os quais têm reduzido nos últimos anos ${ }^{2}$.

Dados oficiais brasileiros evidenciaram que em se tratando das doenças infecciosas e parasitárias, a diarreia figurou como a 1a causa de internação e a 4a etiologia de óbito entre as crianças menores de 5 anos de idade e ainda revelaram que as regiões brasileiras mais afetas foram as menos desenvolvidas2, permitindo inferir que as condições socioeconômicas, de saneamento básico, culturais e políticas 
interferem diretamente no alto índice de doenças diarreicas nesses locais, configurando-se, portanto, como um problema de saúde pública, especialmente em localidades precárias.

Dentre os fatores caracterizados como risco para os episódios diarreicos destacam-se as precárias condições sociossanitárias, visto que o saneamento básico - traduzido no abastecimento de água potável, esgotamento sanitário, limpeza urbana, drenagem e manejo de resíduos - é um dos fatores determinantes da saúde ${ }^{5}$.

Os dados aqui expostos apontaram para as crianças de 1 a 4 anos de idade como aquelas que apresentaram o maior número de internações por diarreia e gastroenterites. 0 acometimento das crianças de maior idade é explicado por Siqueira, Jesus e Camargo ${ }^{6}$ que, ao falarem sobre a vulnerabilidade das crianças a agravos, referem que nesta idade os indivíduos estão em constante descoberta, começam a andar, ter mais liberdade e mais contato com o mundo a partir da manipulação dos objetos e, devido à curiosidade de conhecer o novo sem que possuam a capacidade de prever, evitar e diferenciar situações que as expõem ao perigo, tornam-se vulneráveis a diversas doenças.

Apesar de as crianças de 1-4 anos de idade terem sido as que apresentaram o maior número de internações por diarreia e gastroenterites, as que mais morreram por esta causa foram as menores de 1 ano. Isso se dá pelo fato de que quanto menor for a idade mais frágil é a criança, que se encontra em processo de formação e fortalecimento do sistema imunológico ${ }^{7}$. Além disso, as crianças menores têm maior percentual de água corporal que as crianças de maior idade e maior taxa de água corporal total $(\mathrm{ACT})^{8}$, o que resulta em maiores complicações nos casos de diarreia devido ao maior potencial de serem acometidas pela desidratação e desnutrição.

No que diz respeito ao gênero, houve predominância das crianças do sexo masculino tanto para as internações como para os óbitos. Isto se deve, provavelmente, devido à diferença de comportamento de ambos os sexos e fatores culturais, de forma que os meninos têm maior liberdade de brincar e interagir com o ambiente que as meninas, sendo encorajados a serem mais independentes e participarem de brincadeiras de maior risco, sem supervisão dos adultos em relação às meninas, que recebem maior vigilância dos seus cuidadores ${ }^{9}$.

Ao direcionar a análise por localidade, pode-se constatar que as regiões brasileiras não são afetadas de modo linear, tendo sido o Nordeste a que mais apresentou casos de internações por diarreia e gastroenterites entre as crianças menores de 5 anos de idade no período em questão em todos os anos analisados. O maior número de casos de internações e óbitos por diarreia e gastroenterites na Região Nordeste pode se relacionar ao fato de ser esta a região brasileira em que a fome e a pobreza estão fortemente concentradas, conforme referido pela Organização das Nações Unidas para a Educação, Ciência e Cultura (UNESCO) ${ }^{10}$.

Segundo o Ministério da Saúde $(\mathrm{MS})^{11}$, o risco de morte por diarreia em crianças menores de 5 anos assume uma maior magnitude nas regiões Norte e Nordeste do país, de forma que sua incidência é cerca de quatro a cinco vezes maior que na Região Sul, representando cerca de $30 \%$ do total das mortes entre o período neonatal e o primeiro ano de vida. Ainda sobre essas regiões, o estudo epidemiológico de Bühler $^{12}$ revelou que associado aos óbitos por diarreia infantil os principais indicadores foram os relacionados às condições sociais e demográficas e ao saneamento básico em que elas vivem. Também neste estudo ${ }^{12}$ foi demonstrado que a Região Sul foi a única que não estava relacionada à extrema pobreza associada aos óbitos por diarreia infantil. Tal fato possivelmente resulta das melhores condições de saneamento básico e infraestrutura desta região, conforme divulgado por uma pesquisa realizada pelo Associação Brasileira do Ministério Público do Meio Ambiente ${ }^{13}$ e pelo maior acesso aos serviços de saúde por parte desta população ${ }^{14}$, o que permite uma assistência mais rápida e, por conseguinte, com menor possibilidade de óbito.

A maior incidência tanto das internações como dos óbitos por diarreia e gastroenterites entre crianças negras pode ser associada à vulnerabilidade a que estão expostas estas pessoas, tanto no que concerne ao acesso às condições de saneamento quanto ao acesso aos serviços de saúde. Segundo o $\mathrm{MS}^{3}$, a população negra em 1990 classificada como extremamente pobre era cerca de três vezes maior que a branca, revelando a suscetibilidade destas pessoas às consequências da pobreza.

Salienta-se ainda que a maioria da população negra está concentrada na região Nordeste, local que apresentou maior incidência da diarreia e gastroenterites, evidenciando assim que a região em que elas vivem e as condições precárias de vida desse ambiente contribuem diretamente para este problema.

Convém destacar que houve um número significativo de indivíduos ignorados quanto à variável raça/cor tanto no que diz respeito às internações quanto aos óbitos por diarreia e 
gastroenterites. Tal fato pode mascarar a análise quanto a esta variável, caracterizando-se como um possível viés acerca destes dados. Diante disso, chama-se atenção para a importância de os profissionais de saúde preencherem adequadamente as fichas de notificação de agravos nos serviços de saúde, visto que tais fichas permitem delinear o perfil epidemiológico de determinado agravo, favorecendo o delineamento de um perfil fidedigno e a realização de ações que permitam a promoção da saúde das crianças e prevenção de doenças.

\section{CONCLUSÕES}

Foi observado como panorama da diarreia e gastroenterites entre crianças na última década no Brasil um maior acometimento de indivíduos do sexo masculino, de raça negra, na Região Nordeste. O perfil das internações e óbitos só se diferenciou no que concerne à faixa etária, uma vez que as crianças entre 1 e 4 anos foram as que tiveram maior número de internações, mas aquelas menores de 1 ano foram as que mais morreram por esta causa.

Apesar de ter havido uma queda tanto nos índices de internações como de óbitos em todas as regiões brasileiras, destaca-se que esses números ainda são elevados, especialmente entre crianças mais vulneráveis, como é o caso daquelas de raça negra e que vivem no Nordeste. Tal fato suscita a necessidade da intensificação de ações preventivas e de cuidados às crianças com doença diarreica que habitam aquela região.

\section{Referências}

1. Brasil. Ministério da Saúde. Secretaria de Vigilância em Saúde. Departamento de Vigilância Epidemiológica. Doenças infecciosas e parasitárias: guia de bolso. Brasília, Distrito Federal, 2004. [Citado 2019 mar 16]. Disponível em:

http://bvsms.saude.gov.br/bvs/publicacoes/guia bolso 4ed.pdf

2. Brasil. Ministério da Saúde. DATASUS. [Citado 2019 mar 10]. Disponível em: http://www2.datasus.gov.br/DATASUS/index.ph $\mathrm{p}$

3. Brasil. Ministério do Planejamento, Orçamento e Gestão. Secretaria de Planejamento e Investimentos Estratégicos. Objetivos de Desenvolvimento do Milênio: Relatório Nacional de Acompanhamento. Brasília, Distrito Federal, 2014. [Citado 2019 abr 05]. Disponível em: http://www.ipea.gov.br/portal/images/stories/P DFs/140523_relatorioodm.pdf
4. World Gastroenterology Organisation (WGO). Diarreia aguda em adultos e crianças: uma perspectiva mundial. 2012. [Citado $2019 \mathrm{abr}$ 02]. Disponível em: https://www.worldgastroenterology.org/

5. Teixeira JC, Gomes MHR, Souza JA. Associação entre cobertura por serviços de saneamento e indicadores epidemiológicos nos países da América Latina: estudo com dados secundários. Rev Panam Salud Pública. 2012; 36(6): 419-425.

6. Siqueira SMC, Jesus VS, Camargo CL. Itinerários terapêuticos em situações de urgência e emergência pediátrica em uma comunidade quilombola. Ciência e Saúde Coletiva. 2016; 21(1): 179-189.

7. Diniz LMP, Figueiredo BCG. O sistema imunológico do recém-nascido. Revista Médica de Minas Gerais. 2014; 24(2): 233-240.

8. Sociedade Brasileira de Alimentação e Nutrição. Água, Hidratação e Saúde. 2016. [Citado 2019 abr 05]. Disponível em: http://sban.cloudpainel.com.br/source/Agua-

HidrataAAo-e-SaAde_Nestle_.pdf

9. Fernandes FMFA, Torquato IMB, Dantas MSA, Pontes Júnior FAC, Ferreira JA, Collet N. Queimaduras em crianças e adolescentes: caracterização clinica e epidemiológica. Rev Gaúcha Enferm. 2012; 33(4): 133-141.

10. Organização das Nações Unidas para Educação, Ciência e Cultura (UNESCO). Pobreza e desigualdade no Brasil: traçando caminhos para a inclusão social. 2003. [Citado 2019 abr 05]. Disponível em: http://unesdoc.unesco.org/images/0013/001339 /133974por.pdf

11. Brasil. Ministério da Saúde. Organização Pan-Americana da Saúde. Fundo das Nações Unidas para a Infância. Manual AIDIPI criança: 2 meses a 5 anos. 2017. [Citado 2019 mar 16]. Disponível em: http://portalarquivos.saude.gov.br/images/pdf/2 017/julho/12/17-0095-Online.pdf

12. Bühler HF, Ignotti E, Neves SMAS, Hacon SS. Análise espacial de indicadores integrados determinantes da mortalidade por diarreia aguda em crianças menores de 1 ano em regiões geográficas. Ciência \& Saúde Coletiva. 2014; 19(10): 4131-4140.

13. Associação Brasileira do Ministério Público do Meio Ambiente. Perfil do saneamento básico no Brasil. 2011. [Citado 2019 abr 16]. Disponível em: https://abrampa.jusbrasil.com.br/noticias/28895 42/perfil-do-saneamento-basico-no-brasil

14. Stopa SR, Malta DC, Monteiro CN, SzwarcwaldI CL, Goldbaum M, Cesar CLG. Use of 
and access to health services in Brazil, 2013 National Health Survey. Rev Saude Publica. 2017; 51(Supl 1:3s).

\section{Endereço para Correspondência}

Universidade Federal da Bahia (UFBA)

Av. Dr. Augusto Viana, S/N, Canela. Salvador Bahia.

CEP 40110-060

e-mail: smcsiqueira@hotmail.com

Recebido em 07/05/2020

Aprovado em 02/03/2021

Publicado em 31/03/2021 